\title{
COVID-19 Trends in the Phoenix Metropolitan Area from a Mobile Testing Program: Last Quarter of 2020
}

\author{
Christine S. M. Lau ${ }^{1} \cdot$ Sebastian Shu ${ }^{1} \cdot$ Jennifer Mayer ${ }^{1} \cdot$ Mikayla Towns $^{1} \cdot$ Alexis Farris $^{1} \cdot$ Felissa Washington $^{2}$. \\ Pablo Prichard ${ }^{1} \cdot$ Vershalee Shukla ${ }^{1}$
}

Accepted: 19 April 2021 / Published online: 29 April 2021

(c) The Author(s), under exclusive licence to Springer Science+Business Media, LLC, part of Springer Nature 2021

\begin{abstract}
The coronavirus (COVID-19) pandemic continues to be a public health concern, and Arizona has once again been a COVID19 hotspot, peaking at 118.3 cases per 100,000. Understanding the trends in COVID-19 positivity rates over time is crucial in planning and mitigation of the virus. This current study analyzes the trends in COVID-19 testing, and COVID-19 antigen and antibody positivity rates over a 3-month time-span from October to December 2020. A retrospective study was conducted collecting data from a mobile testing program during October to December 2020 in the Phoenix metropolitan area. COVID-19 antigen and antibody positivity rates were analyzed. A total of 6710 patients were included in the study. As the months progressed, more patients were tested (October: 1635; November: 2037; December: 3038). The COVID-19 antigen positivity rate was significantly higher in December, compared to October and November (13.43\% vs. 11.43 and $10.86 \%$, $\mathrm{p}=0.021)$. COVID-19 IgG rates were also significantly higher in November and December, compared to October $(16.65$ and $16.50 \%$ vs. $8.74 \%, \mathrm{p}<0.001$ ). There was a progressive increase in COVID-19 positivity cases towards the end of 2020 , likely attributed to factors including social gatherings during the holidays and the relaxing of the closure restrictions. Continued public health measures is crucial in preventing the spread of COVID-19.
\end{abstract}

Keywords COVID-19 $\cdot$ SARS-CoV-2 $\cdot$ Mobile testing $\cdot$ Phoenix $\cdot$ Arizona

\section{Introduction}

One year since the beginning of the global outbreak of the coronavirus (COVID-19), the COVID-19 pandemic continues to be a public health challenge. By the end of 2020 , there was nearly 84 million cases reported worldwide and 20 million cases in the United States [1]. In Arizona, the first case was reported on January 22, 2020 [2], and within 2020 alone, there were 520,000 cases and 8800 deaths according to the Arizona Department of Health Services (ADHS) [3]. Arizona has even been declared a COVID-19 hotspot of the world with 118.3 new cases per 100,00 people [4].

Pablo Prichard and Vershalee Shukla are Co-senior authors.

Christine S. M. Lau

drlau@vincerecancer.com

1 Vincere Cancer Center, 7469 E. Monte Cristo Avenue, Scottsdale, AZ 85260, USA

2 City of Phoenix, Phoenix, AZ, USA
The State of Arizona declared a state of emergency on March 11, 2020 in attempts to reduce the spread of COVID19. Several restrictions to limit crowds and gatherings followed. Via executive orders many businesses were mandated to close including restaurant dining areas (only allowing take-out service), bars, indoor gyms, fitness clubs, and movie theaters [5] Other measures including social distancing, stay-at-home orders, and enhanced sanitation in private and public spaces were also implemented. The use of face masks in public areas were also recommended. These public health measures have been extensively supported by scientific evidence. In a systematic review and meta-analysis by Chu et al., using face masks was associated with a $14.3 \%$ risk reduction of getting COVID-19 infection or virus transmission compared to not using face masks [6]. Data also showed that social distancing of at least $1 \mathrm{~m}$ versus less than $1-\mathrm{m}$ decreased chances of virus transmission by $10.2 \%$ (95\% CI of $-11.5 \%$ to -7.5$)$. Translating social distancing to a bigger scale, in a study involving 149 countries, social distancing interventions were associated with a $13 \%$ reduction in the incidence of COVID-19 (95\% CI of 0.85-0.89) [7]. 
The effect of public health measures implemented by the State of Arizona helped contain the initial phase of the first surge of COVID-19 cases. As a result of the lower number of cases, Arizona began relaxing the restrictions and reopening the State. On March 31, 2020, the State established 21 policies which kept the 7-day average cases below 300, while deaths remained below 15 [8]. Later in May with the executive order 2020-36: "Stay Healthy, return smarter, return stronger" was announced and the state partially re-opened businesses with COVID-19 guidelines [9]. Relaxing public health measures led to a gradual increase in COVID-19 cases that peaked after Independence Day (July 4th), reaching a 7-day average peak of 3849 cases and 80 deaths [8]. Additionally, schools restarted in-person activities in July via executive order 2020-51 [10]. As a measure of control, the ADHS published benchmarks to orient the decision making of keeping in-person learning activities based on COVID-19 cases, COVID-like illness, and positivity rate per county [11].

Recently, Arizona experienced a second surge of cases that surpassed the first wave. Understanding the trends in testing and positivity rates over time is crucial in planning and mitigation of the virus. This current study analyzes the trends in COVID-19 testing, and COVID-19 antigen and antibody positivity rates over a 3-month time-span from October to December 2020.

\section{Methods}

A retrospective study was conducted collecting data from a mobile testing program during October to December 2020 in the Phoenix metropolitan area. Vincere, in partnership with the City of Phoenix, operated a mobile COVID-19 testing site offering COVID-19 antigen and antibody testing. Testing sites varied on a daily basis and were chosen in collaboration with the City of Phoenix based on reported case counts and access to testing and diagnosis. Often, these included highly dense areas around the city with limited resources. Individuals of 12 years old or older had the option to schedule an appointment or do a walk-up visit. Children under 12 years old were excluded from testing and suggested to contact their primary care provider or pediatrician. Testing was offered at zero cost to the patient and patients without insurance were eligible to receive testing at no cost as well.

\section{COVID-19 Antigen and Antibody Test}

Both the antigen and antibody tests utilized were Food and Drug Administration (FDA) Emergency Use Authorization (EUA) approved tests [12]. All tests were performed by certified emergency medical technicians, paramedics, registered nurses, or medical doctors. All results were read and interpreted by healthcare professionals and given to patients onsite within $20 \mathrm{~min}$.

\section{Antigen Test}

The Quidel SOFIA ${ }^{\circledR}$ SARS antigen fluorescent immunoassay was utilized. This qualitative test uses immunofluorescence to detect the nucleocapsid protein from SARS-CoV-2 in nasal or nasopharyngeal samples. Every SOFIA ${ }^{\circledR}$ machine was calibrated each day before use. This test has a sensitivity of $80 \%$ and a specificity of $98.9 \%$, and a positive predictive accuracy of $94.1 \%$ and negative predictive accuracy of $95.9 \%[13]$

\section{Antibody Test}

Antibody testing was performed using the Assure COVID$19 \mathrm{IgG} / \mathrm{IgM}$ Rapid Test, a rapid lateral flow chromatographic immunoassay which qualitatively detects and differentiates IgM and IgG antibodies to SARS-CoV-2. This FDA EUA test has a combined sensitivity of $100 \%$ and specificity of $98.8 \%$ [12]. Using a finger prick, a small blood sample was extracted and diluted in the cassette.

\section{Outcomes}

Primary outcome: COVID-19 antigen positivity rate.

Secondary outcome: COVID-19 IgG positivity rate, COVID-19 antigen positive patient symptoms.

\section{Statistical Analysis}

One week worth of data from each month, spaced four weeks apart, was extracted. Dates included October 10-16, November 7-13, and December 5-11, 2020. Participant data was grouped into three groups-October, November, and December. Further analysis was stratified using COVID-19 positive and COVID-19 negative antigen data. Categorical data was analyzed using Chi-square test and continuous data were analyzed with Student's $t$-test and analysis of variance (ANOVA). All statistical analysis was conducted using IBM SPSS $® v 25$. Statistical significance was accepted at a level of $\mathrm{p}<0.05$.

\section{Results}

A total of 6710 patients were included in the study. As the months progressed, more patients were tested (October: 1635; November: 2037; December: 3038) (Table 1). Average age was 39.78 years (October: 41.40 years; November: 
40.19 years; December 38.64 years). Male-to-female ratio was 0.84 (October: 0.96; November: 0.82; December: 0.86).

COVID-19 antigen positivity rate was significantly higher among December, compared to October and November $(13.43 \%$ vs. $11.43 \%$ and $10.86 \%, p=0.021)$ (Table 2 ). COVID-19 IgG rates were also significantly higher among November and December, compared to October $(16.65 \%$ and $16.50 \%$ vs. $8.74 \%, \mathrm{p}<0.001)$.

Patients who tested positive for COVID-19 antigen were younger (38.47 years vs. 40.00 years), compared to those who tested negative $(p<0.01)$. A higher percentage of males tested positive for COVID-19 antigen compared to females $(18.34 \%$ vs. $15.58 \%, p=0.032)$. Approximately $63.68 \%$ of patients who tested positive for the virus were symptomatic, with the most common symptoms being cough (30.79\%), headache $(24.21 \%)$, and fever (18.42\%). Among those who tested COVID-19 antigen positive, patients who developed symptoms were slightly younger than those who were asymptomatic (36.36 years vs. 38.29 years, $p<0.01$ ) but there was no significant difference among gender (males: $62.56 \%$ vs. females: $64.86 \%, p=0.64$ ).

\section{Discussion}

This retrospective observational study reports the antigen and antibody results from a mobile testing site during the last quarter of 2020 in Phoenix, the most densely populated city in the state of Arizona. Phoenix, situated in Maricopa county, contains the largest number of COVID-19 cases in the state which represent $62.2 \%$ of all cases reported in Arizona (as of January 26, 2021). Maricopa also ranks second in the US for the county with the most COVID-19 cases $[1,3]$.

The results of this study show a progressive increment of COVID-19 cases and positivity rates towards the end of 2020, which is consistent with the findings reported by the Arizona Department of Health Services (ADHS) [3]. This uptrend could be attributed to several factors, including social gatherings during the holidays and the relaxing of the closure restrictions. There were several holidays between October and December 2020 which congregated large numbers of people, that could have provoked a "snowball effect": Thanksgiving (November 26th) and December holidays including Hanukkah and Christmas. These holidays, which are a time of family gatherings and celebrations, increased the chances of close contact. Furthermore, air travel increased during this time, with many airports having large crowds of passengers waiting for flights. Despite a $60 \%$ reduction of travelers compared to 2019, Thanksgiving weekend (from November 26th to the 29th) alone, mobilized 4.5 million people in airports across the country according to the Transportation Security Administration [14]. In Japan, a contact tracing study concluded that closed environment events had 18 times more risk of transmission than open environment events [15].

The uptrend could also be explained by the relaxing of some mandates and the re-opening of the state. Arizona has been partially open since May 17, 2020, allowing businesses to restart activities with limited capacity [8]. Since the reopening, no major social restrictions mandates have been enforced. Weddings, funerals, religious and sport events have been recognized as high risk for transmission of COVID-19 [16-18]. Chande et al. have developed a COVID19 event risk assessment planner to evaluate the probability of having one or more COVID-19 positive cases in the event based on the number of people attending and the incidence rate reported by the state [19]. For example, if a wedding with 50 people is organized in Maricopa country, the risk assessment calculator estimates a $57 \%$ chance of having one person with COVID-19.

Another factor that might have influenced the positivity rate could be the location of the testing sites. The testing site was chosen intentionally to assist the underserved population in Maricopa county. Many of these neighborhoods have a high Latino and African American population-the two races combined represent $38.8 \%$ of the population of Arizona [20]. High incidence and severity of COVID-19 have been reported disproportionately in ethnic/racial minorities. In an ecological study in the three most populous counties in each US state and territory, it was found that for every $1 \%$ of African-American density there was a $5 \%$ increase in COVID-19 prevalence $(\mathrm{p}<0.01)$ and an increase of 2 deaths due to COVID-19 per 100,000 population $(\mathrm{p}=0.02)$ [21]. Similarly, Rodriguex-Diaz et al. reported that in highly dense Latino communities ( $\geq 17.8 \%$ of the population) in the Northeast and Midwest regions, the rate of COVID-19 cases was higher (adjusted relative risk: $1.42,95 \%$ CI 1.11-1.84 and $1.70,95 \%$ CI $1.57-1.85$, respectively) than less densely Latino communities ( $<17.8 \%$ of the population) [22].

The uptrend in the study could be also explained by the appearance of molecular variants of the virus. For example, in Houston, Texas, a variant with Gly614 amino acid mutation in the spike protein was detected and reported to have increased infectivity capacity due to more stability. As a result, their first wave had $82 \%$ of this variant while the second wave had $99.9 \%(p<0.0001)$ [23].

The largest age group of COVID-19 cases found in this study were young adults between 22 and 40 years old which concurs with national data [24]. Even before the study period, the CDC reported in September a switch of tendency of age group affected; changing the median age from 46 years in May 2020 to 37 years in July 2020 and 38 years in August 2020 [25]. In another publication from a national reference laboratory, it reported in March to April a median age of 40.8 years (Interquartile range (IQR) 
29.0-54.1) and 35.8 years (IQR 24.0-50.2) in June to July [26]. Several factors, including the start of college semesters and in-class education, may have played a role in the increase in COVID-19 cases among young adults. With our study, it reinforces the idea of taking more protective measures towards younger adults before a COVID-19 surge. This might allow to decrease the speed of transmission to earn more time for vaccination.

This study has several limitations. The SOFIA ${ }^{\circledR}$ Antigen test by Quidel (San Diego, USA) does not have the power to differentiate SARS-CoV and SARS-CoV-2 as the antigen used for detection is highly conservative among Coronaviruses [27]. According to the CDC however, there are no current reported cases of SARS-CoV, and all reported SARS$\mathrm{CoV}$ positive results should be communicated immediately to authorities [28]. Another limitation of this study is sample selection. This study is mostly representative of the dense urban metropolitan area, especially the underserved areas of Phoenix which have larger Latino and African American populations, and low-income communities. Furthermore, since the mobile testing site was available at zero-cost to the patient, individuals are more likely to get tested, allowing for the detection of asymptomatic cases.

In the future, more efforts need to be done in reporting the epidemiology of COVID-19 in rural areas and in racial minorities such as the Indian tribes. Access to healthcare in American Indian tribes have been reported to be chronically neglected and currently exposed to a rapid COVID19 outbreak in the reservations $[29,30]$. This is relevant given the fact that since November 2, 2020, the majority of COVID-19 cumulative cases in Arizona were located in non-metropolitan area [24]. Additionally, enhancing testing among the population could lower anxiety and improve work-life balance as described in first responders [31]. This evidence reinforces the importance of testing and mitigation strategies in Arizona.

\section{Conclusion}

The last quarter of 2020 ended up with an uptrend of COVID-19 cases and an increase in COVID-19 positivity rates. Several factors, including holiday gatherings, the relaxing of the closures and reopening of the state, and the start of school semesters and in-classroom education, likely all contributed to the increase in COVID-19 rates. Further epidemiological studies are needed to understand the status of COVID-19 in non-metropolitan areas of Arizona and racial minorities as well as action needs to be taken towards protecting young adults in the metropolitan area of Maricopa county.
Table 1 Characteristics of people tested at the COVID-19 mobile program during last quarter of 2020

\begin{tabular}{lllll}
\hline & October & November & December & P value \\
\hline N (total=6710) & 1635 & 2037 & 3038 & \\
Age, average (years) & 41.40 & 40.19 & 38.64 & $<0.001$ \\
Under 20 years (\%) & 10.6 & 11.6 & 9.9 & \\
20-44 years (\%) & 47.9 & 49.2 & 55.4 & \\
45-54 years (\%) & 16.5 & 16.6 & 17.0 & \\
55-64 years (\%) & 14.2 & 12.1 & 11.2 & \\
65 years and over (\%) & 10.9 & 10.5 & 6.6 & \\
Gender & & & & 0.684 \\
Male & 48.98 & 44.96 & 46.21 & \\
Female & 51.02 & 55.04 & 53.79 & \\
M:F ratio & 0.96 & 0.82 & 0.86 & \\
\hline
\end{tabular}

$N$ number, $M: F$ male to female

Table 2 COVID-19 antigen and antibody results

\begin{tabular}{llllr}
\hline & October & November & December & P value \\
\hline N (total=6710) & 1635 & 2037 & 3038 & \\
Antigen positive (\%) & 11.43 & 10.86 & 13.43 & 0.021 \\
IgM positive (\%) & 9.40 & 8.72 & 8.66 & 0.473 \\
IgG positive (\%) & 8.74 & 16.65 & 16.50 & $<0.001$ \\
\hline
\end{tabular}

$N$ number

Acknowledgements We would like to thank the City of Phoenix for their partnership with the mobile COVID-19 testing project. We would also like to thank Ira Puranik, Hurshneet Chadha, and Addison Berger for their help with data extraction. Furthermore, this mobile COVID19 testing program would not have been possible without the help of all the individuals who help operate the site each day and perform the testing.

Funding The mobile COVID-19 testing program was done in partnership with the City of Phoenix.

\section{Declarations}

Conflict of interest The authors have no conflict of interest to declare.

\section{References}

1. COVID-19 Map [Internet]. Johns Hopkins Coronavirus Resource Center. Retrieved January 3, 2021, from https://coronavirus.jhu. edu/map.html

2. Scott, S. E., Zabel, K., Collins, J., Hobbs, K. C., Kretschmer, M. J., Lach, M., Turnbow, K., Speck, L., White, J. R., Maldonado, K., \& Howard, B. (2020). First mildly ill, non-hospitalized case of coronavirus disease 2019 (COVID-19) without viral transmission in the United States-Maricopa County, Arizona, 2020. Clinical Infectious Diseases, 71(15), 807-812. 
3. AZDHS I COVID-19 Dashboards [Internet]. Arizona Department of Health Services. Retrieved January 1, 2021, from http://www. azdhs.gov/preparedness/epidemiology-disease-control/infectiousdisease-epidemiology/covid-19/dashboards/index.php

4. An interactive visualization of COVID-19/91-DIVOC [Internet]. Retrieved January 18, 2021, from https://91-divoc.com/pages/ covid-visualization/

5. Gallaway, M. S., Rigler, J., Robinson, S., Herrick, K., Livar, E., Komatsu, K. K., Brady, S., Cunico, J., \& Christ, C. M. (2020). Trends in COVID-19 incidence after implementation of mitigation measures-Arizona, January 22-August 7, 2020. Morbidity and Mortality Weekly Report, 69(40), 1460-1463.

6. Chu, D. K., Akl, E. A., Duda, S., Solo, K., Yaacoub, S., Schünemann, H. J., et al. (2020). Physical distancing, face masks, and eye protection to prevent person-to-person transmission of SARSCoV-2 and COVID-19: A systematic review and meta-analysis. The Lancet, 395(10242), 1973-1987.

7. Islam, N., Sharp, S. J., Chowell, G., Shabnam, S., Kawachi, I., Lacey, B., Massaro, J. M., D'Agostino, R. B., \& White, M. (2020). Physical distancing interventions and incidence of coronavirus disease 2019: Natural experiment in 149 countries. BMJ, 370, $\mathrm{m} 2743$.

8. Georgetown University Center for Global Health Science and Security. COVID analysis and mapping of policies-Arizona [Internet]. COVID AMP. Retrieved January 22, 2021, from https:// covidamp.org/model\#AZ

9. Office of Governor Doug Ducey I Executive Orders [Internet]. Office of the Arizona Governor. Retrieved January 18, 2021, from https://azgovernor.gov/executive-orders

10. Ducey, D. (2020). Executive Order 2020-51: "Arizona Open for Learning" [Internet]. State of Arizona. Retrieved from https:// azgovernor.gov/executive-orders

11. Arizona Department of Health Services. (2020). Safely returning to in-person instruction [Internet]. Retrieved from https://www. azdhs.gov/documents/preparedness/epidemiology-disease-contr ol/infectious-disease-epidemiology/novel-coronavirus/covid-19safely-return-to-in-person-instruction.pdf

12. Health C for D and R. (2021). EUA Authorized Serology Test Performance. FDA [Internet]. Retrieved January 22, 2021, from https://www.fda.gov/medical-devices/coronavirus-disease-2019covid-19-emergency-use-authorizations-medical-devices/euaauthorized-serology-test-performance

13. Pray, I. W. (2021). Performance of an antigen-based test for asymptomatic and symptomatic SARS-CoV-2 testing at two university campuses-Wisconsin, September-October 2020. Morbidity and Mortality Weekly Report, 69. Retrieved January 9, 2021, from https://www.cdc.gov/mmwr/volumes/69/wr/mm695 152a3.htm

14. Transportation Security Administration. TSA checkpoint travel numbers (current year(s) versus prior year/same weekday) I Transportation Security Administration [Internet]. Retrieved January 22, 2021, from https://www.tsa.gov/coronavirus/passenger-throu ghput

15. Nishiura, H., Oshitani, H., Kobayashi, T., Saito, T., Sunagawa, T., Matsui, T., Wakita, T., MHLW COVID-19 Response Team, Suzuki, M. (2020). Closed environments facilitate secondary transmission of coronavirus disease 2019 (COVID-19). medRxiv. https://doi.org/10.1101/2020.02.28.20029272

16. Leclerc, Q. J., Fuller, N. M., Knight, L. E., Funk, S., \& Knight, G. M. (2020). What settings have been linked to SARS-CoV-2 transmission clusters? Wellcome Open Research. https://doi.org/ 10.12688/wellcomeopenres.15889.2

17. Memish, Z. A., Steffen, R., White, P., Dar, O., Azhar, E. I., Sharma, A., \& Zumla, A. (2019). Mass gatherings medicine: Public health issues arising from mass gathering religious and sporting events. The Lancet, 393(10185), 2073-2084.
18. Sassano, M., McKee, M., Ricciardi, W., \& Boccia, S. (2020). Transmission of SARS-CoV-2 and other infections at large sports gatherings: A surprising gap in our knowledge. Frontiers in Medicine, 7, 277.

19. Chande, A. T., Gussler, W., Harris, M., Lee, S., Rishishwar, L., Hilley, T., Jordan, I. K., Andris, C. M., \& Weitz, J. S. (2021). COVID-19 event risk assessment planning tool [Internet]. Retrieved January 26, 2021, from https://covid19risk.biosci.gatech.edu/

20. U.S. Census Bureau QuickFacts: Maricopa County, Arizona [Internet]. Retrieved January 2, 2021, from https://www.census. gov/quickfacts/fact/table/AZ, maricopacountyarizona

21. Cyrus, E., Clarke, R., Hadley, D., Bursac, Z., Trepka, M. J., Dévieux, J. G., Bagci, U., Furr-Holden, D., Coudray, M., Mariano, Y., \& Kiplagat, S. (2020). The impact of COVID-19 on African American communities in the United States. Health Equity, 4(1), 476-483.

22. Rodriguez-Diaz, C. E., Guilamo-Ramos, V., Mena, L., Hall, E., Honermann, B., Crowley, J. S., Baral, S., Prado, G. J., MarzanRodriguez, M., Beyrer, C., \& Sullivan, P. S. (2020). Risk for COVID-19 infection and death among Latinos in the United States: Examining heterogeneity in transmission dynamics. Annals of Epidemiology, 52, 46-53.e2.

23. Long, S. W., Olsen, R. J., Christensen, P. A., Bernard, D. W., Davis, J. J., Shukla, M., Nguyen, M., Saavedra, M. O., Yerramilli, P., Pruitt, L., Subedi, S. (2020). Molecular architecture of early dissemination and massive second wave of the SARS-CoV-2 virus in a major metropolitan area. medRxiv [Internet]. Retrieved January 26, 2021, from https://www.ncbi.nlm.nih.gov/pmc/articles/ PMC7536878/

24. CDC. (2020). COVID-19 cases, deaths, and trends in the US I CDC COVID Data Tracker [Internet]. Centers for Disease Control and Prevention. Retrieved January 3, 2021, from https://covid.cdc. gov/covid-data-tracker

25. Boehmer, T. K. (2020). Changing age distribution of the COVID19 pandemic-United States, May-August 2020. MMWR: Morbidity and Mortality Weekly Report, 69, 759-765.

26. Greene, D. N., Jackson, M. L., Hillyard, D. R., Delgado, J. C., $\&$ Schmidt, R. L. (2020). Decreasing median age of COVID-19 cases in the United States-Changing epidemiology or changing surveillance? PLoS ONE, 15(10), e0240783.

27. Lin, D., Liu, L., Zhang, M., Hu, Y., Yang, Q., Guo, J., Dai, Y., Xu, Y., Cai, Y., Chen, X., \& Huang, K. (2020). Evaluations of the serological test in the diagnosis of 2019 novel coronavirus (SARS-CoV-2) infections during the COVID-19 outbreak. European Journal of Clinical Microbiology \& Infectious Diseases, 39(12), 2271-2277.

28. CDC. SARS Surveillance guide [Internet]. Retrieved February 10, 2021, from https://www.cdc.gov/sars/guidance/b-surveillan ce/reporting.html

29. Chhabra, A., Sood, V., Sood, V., \& Sood, A. (2020). Improving testing for COVID-19 for the rural Southwestern American Indian Tribes. Southwest Journal of Pulmonary and Critical Care, 20(5), 175-178.

30. Kakol, M., Upson, D., \& Sood, A. (2020). Susceptibility of Southwestern American Indian Tribes to coronavirus disease 2019 (COVID-19). The Journal of Rural Health. https://doi.org/10. $1111 /$ jrh. 12451

31. Shukla, V., Lau, C. S. M., Towns, M., Mayer, J., Kalkbrenner, K., Beuerlein, S., \& Prichard, P. (2020). COVID-19 exposure among first responders in Arizona. Journal of Occupational and Environmental Medicine, 62(12), 981-985.

Publisher's Note Springer Nature remains neutral with regard to jurisdictional claims in published maps and institutional affiliations. 